Apidologie, 1977, 8 (4), 321-335.

\title{
NEKTARIEN UND IHRE ÖKOLOGISCHE BEDEUTUNG
}

\author{
Les nectaires et leur signification écologique
}

\author{
Stefan VOGEL \\ Botanisches Institut und Botanischer Garten \\ Universität Wien \\ WIEN (Österreich)
}

\author{
SUMMARY \\ NECTARIES AND THEIR ECOLOGICAL SIGNIFIGANCE
}

This review summarizes our present knowledge and open questions about various aspects of the nectaries : their morphology, topography, distribution in the plant kingdom, and ecological significance, including extranuptial nectaries and some exceptional cases. Progressive trends in nectary evolution and the different ways by which animals utilize the nectar are also discussed.

\section{ZUSAMMENF ASSUNG}

Das Referat gibt einen kurzgefaßten Übexblick über Morphologie, Topographie, Verbreitung und ökologische Funktion der Nektardrüsen samt ihrer Problematik in heutiger Sicht, unter Einschluß der extranuptialen Nektarien und einiger lehrreicher Sonderfälle. Besprochen werden ferner die Progressionsrichtungen der Nektarienevolution und die verschiedenen Arten der Nutzung des Nektars durch Tiere.

Nektar und Ambrosia, Trank und Speise in den Symposien des Olymp, gaben in unserer Wissenschaft bekanntlich die Namen ab für zwei eßbare Naturprodukte, die beide eine Rolle in den Beziehungen zwischen Pflanzen und Tieren spielen : Ambrosia wurde die von Borkenkäfern und bestimmten Ameisen gezüchtete Pilznahrung genannt, und unter Nektar versteht man jenes zuckerhaltige Sekret, das unter den Anlockungsmitteln der Blüten die 
erste Stelle einnimmt. Laien verbinden dieses Stichwort vor allem mit der Honigbiene, die diesen Blütensaft saugt, und mit Honig, den sie aus ihm bereitet.

Dieses Referat will Ausschau halten und deutlich machen, daß das eben genannte Geschehen - trotz seiner Bedeutung für den Menschen - doch nur Einzelfall eines vielgestaltigen biologischen Phänomens ist. Nektar und Nektarien sind ja nicht auf Blüten beschränkt, und Nektar ist weit mehr als nur Bienenfutter. Allerdings muß es genügen, den Rahmen durch ein paar lehrreiche und auch extreme Beispiele abzustecken, die die Stellung der Nektarien im Vegetationskörper und ihre ökologische Rolle kennzeichnen.

\section{EXKRETIONSORGAN ODER LOCKINSTRUMENT ?}

Wenige Worte mögen über ein in der Nektarienliteratur verbreitetes erkenntnistheoretisches Dilemma vorausgeschickt werden. Ein Teil der Autoren vertritt den Standpunkt, nur die physiologische Erklärung der Nektarsekretion sei die einzig exakte, ihre ökologische Bedeutung nur zufällig und nicht genau faßbar. Andere sehen sie dagegen allein als umweltgezüchtetes Anpassungsprodukt an. Nicht selten werden physiologisch-kausale und teleologisch-finale Argumentationen unbedacht vermengt. Wir ptlichten jener Ansicht bei, die beide Aspekte für wissenschaftlich legitime Fragestellungen hält, die keine sich ausschließenden Alternativen darstellen, sondern in jedem konkreten Fall, auch wenn er in Wirklichkeit eine Einheit ist, beide, und zwar in logischer Trennung, geprüft werden müssen.

Bei manchen Typen von Nektarien tritt deren ätiologische Bedingtheit freilich absolut in den Vordergrund, bei anderen gerade ihre Umweltbeziehung. Das heißt, einmal erscheint Nektar mehr als Exkret (um die zoophysiologische Terminologie der Abscheidungen zu gebrauchen), ein andermal mehr als ein synökologisch bedeutsames Sekret. Auch wäre es verfehlt, für jede minimale Exsudation zuckerhaltiger Säfte einen exotropen Nutzen zu postulieren oder andererseits etwa die Blütennektarien als eine physiologische Notwendigkeit hinstellen zu wollen. Der Physiologe kann hier nur untersuchen, warum ein solcher Sekretionsapparat im Rahmen des Stoffwechsels einer bestimmten Pflanze angelegt bzw. möglich ist, wie er zu instrumenteller Nutzanwendung z.B. im Dienste der Bestäubung gesteigert werden konnte und weshalb er bei anderen Pflanzen fehlt. Die Gegenwart oder das Fehlen von Nektarien ist auch ein Bauplanmerkmal. Manche Pflanzen bedürfen ihrer offenbar, um in der Laubregion zu exzernieren, andere haben - selbst in den Blüten - grundsätzlich keine. Wieder andere führen im Laub Nektarien, aber in den Blüten nicht und umgekehrt. 
Nektarien treten, wie oben angedeutet, in einer breiten Skala von diffuser, anatomisch kaum erkennbarer Abscheidung bis zu hochentwickelten Apparaten auf; auch an der gleichen Pflanze kommt ein Nebeneinander ökologisch irrelevanter Laubdrüsen und komplizierter Blütenlockorgane vor.

\section{VERBREITUNG IM PFLANZENREICH}

Überblicken wir zunächst kurz, in welchen Hauptgruppen der Pflanzen sich Nektarausscheidung findet! Bei Pilzen (Puccinia graminis, Claviceps purpurea) tritt sie erstmals auf, schon hier bekanntlich im Dienst entomophiler Spermatien- bzw.Konidienverbreitung dieser Pflanzenparasiten. Ob die honigtauartige Abscheidung wirklich ein Pilzprodukt oder nicht vielleicht eine lokale pathologische, wenn auch fremddienliche Reaktion des Wirtes ist, bleibt indessen noch zu prüfen. An Farn-Wedeln kommen Drüsen vor, die extraflorale Nektarien genannt werden könnten, wenn dieser Begriff hier einen Sinn hätte. Pteridium aquilinum (Schremmer 1969) und viele tropische Polypodiaceen führen sie. Den Gymnospermen fehlen Nektarien; nur als Befruchtungstropfen tritt Zuckersaft bei einigen Familien aus der Mikropyle hervor, wohl ein durch osmotische Wasseraufnahme verdünntes Produkt des zerfallenden Nucellusscheitels. Er dient als Auffänger und Keimmedium des Pollens. Bei den Gnetophyten kann die Abscheidung jedoch zugleich im Rahmen einer primitiven Entomophilie als Lockmittel fungieren. In den Blüten der Bedecktsamer übernehmen Narbensekrete die Hauptfunktion des Befruchtungstropfens. Sie sind ebenfalls oft zuckerhaltig, spielen aber nur vereinzelt zugleich auch die Rolle eines Beköstigungsmittels (Drymis, Akebia, Araceen). Diese Aufgabe besorgen bei den meisten Angiospermen die echten Nektarien, Organe, die weder auf die Blüte beschränkt noch durchweg Lockmittel sind. Vor allem finden sie sich auf den Blättern, mit Ausnahme des Blütendiscus nur selten auf der Sproßachse. Den Wurzeln fehlen sie und so gut wie immer den Früchten.

Ihre anatomische Verschiedenheit (umfassend behandelt von Zimmermann 1932, Feldhofen 1933, Brown 1938, Daumann 1970) zeugt von polyphyletischer Entstehung. Bei den sogenannten gestaltlosen Nektarien ist eine drüsige Zelldifferenzierung nicht erkennbar. Ihr Sekret entsteht im Mesophyll, tritt über die Interstitien durch die Stomata aus. Ansonsten haben nectarogene Zellkomplexe die histologischen und zytologischen Merkmale einer Drüse. Ohne ins Einzelne zu gehen, wollen wir sie im Interesse des folgenden grob in mesophylläre, epitheliale und trichomatische Nektarien einteilen. Bei ersteren ist das innere Blatt-oder Achsengewebe drüsig, der Nektar entweicht meist durch mehr minder verschlußunfähig gewordene Spaltöffnungen (Saftspalten; ZandonELLA 1967). Epithelnektarien bestehen 
aus einer direkt abscheidenden, palisadenartigen, oft von weiteren Drüsenschichten unterlagerten Epidermis. Trichomatische Nektarien sind Drüsenhaarfluren und damit ebenfalls epidermaler Natur. Bei Bignoniaceen, Cucurbitaceen u.a. können auch einzelnstehende Haare durch anticlinale Teilungen so verbreitert sein, daß sie wie schüsselförmige Epithelnektarien aussehen (Zimmermaxn 1932). Selten entstehen Nektarien aus Abscissions-Flächen, so aus denen früh abfallender Blütenanlagen und Infloreszenzgipfel einiger Leguminosen (Apios, Canavalia; Foerste, nach KNuTH 1904) oder von Brakteen (bei der Labiatengattung Becium).

Zwischen nectarogenen Zellkomplexen und solchen, die andere Substanzen sezernieren, bestehen oft enge histogenetische und damit organophyletische Beziehungen, selbst innerhalb derselben Pflanzenart. Hydathoden können sich zu Nektarien umgewandelt haben, wie bei Papilionaceen und den Malvales, oder Nektarien haben sich zu Öldrüsen transformiert (so bei einigen Gattungen der Malpighiaceen, Iridaceen, Scrophulariaceen; Voges 1974). Solche Organbeziehungen sind noch wenig bekannt und versprechen besonders in ihrem feinstrukturellen Aspekt aufschlußreiche Ergebnisse.

\section{ÖKOSYSTEMATIK UND TOPOGRAPHIE DER NEKTARIEN}

Beim Versuch einer kurzgefaßten ökosystematischen Übersicht der Nektarien wollen wir zunächst festhalten, daß sie bei den Angiospermen in zwei Hauptversionen auftreten : als florale und extraflorale Nektarien. LiNNÉ hatte mit Nektar nur das Blütensekret gemeint (1735); Nektarien nannte er die es absondernden ganzen Blätter. SPRENGEL unterschied dann zwischen der eigentlichen Drüse und dem das Sekret bergenden Behälter, dem ( Safthalter ) (Nectarotheca). Obwohl die Speisefunktion des Nektars, wie schon bemerkt, im vegetativen Bereich weniger eindeutig ist, wurden dann auch die vegetativen Drüsen als Nektarien bezeichnet (CASPARY 1848). Nektarien gibt es also entweder an bzw. in den Blüten oder aber außerhalb davon. Im allgemeinen deckt sich diese topographische Unterscheidung auch mit der ökologischen. Die floralen Nektarien sind in die Bestäubung eingeschaltet, die extrafloralen nicht.

Manche Nektarien an Blüten haben jedoch keine blütenökologische Funktion, z.B. die auf der Außenseite des Perigontubus einer Reihe von Iris-Arten oder auf der Coroll-Rückseite zahlreicher Bignoniaceen, die anatomisch und funktionell zu den extrafloralen Nektarien* gehören. Umge-

* Der Ausdruck wird heute im Sinne CASPary's gebraucht. Delpivo verstand unter extrafloralen Nektarien die biologisch mit der Blütenfunktion verknüpften (nuptialen), aber außerhalb der Blüte stehenden Nektarien. 
kehrt sind hier und da Nektarien, die morphologisch vegetative Drüsen sind, zu Blütenlockorganen geworden.

Despino unterschied wegen dieser Überschneidungen extranuptiale und nuptiale Nektarien, wörtlich solche, die bei der Pflanzenhochzeit mitwirken, und sol̉che ohne diese Rolle. Die erwähnten Drüsen der Schwertlilie wären demnach zwar floral, aber extranuptial. Ihnen dicht benachbart liegt im Tubus-Innern ein zweites, nuptiales System, das sich in diesem Fall auch darin unterscheidet, daß es zusätzlich Rohrzucker sezerniert, der im Sekret des extranuptialen Nektariums fehlt (z.B. bei Iris fulva, Vogec 1967). Umgekehrt sind etwa die Drüsen der Euphorbia-Cyathien extrafloral, aber nuptial. Ganz selten ist ein florales Nektarium ökologisch ambivalent. So sind die paarigen Mündungen des Septalnektariums der Haemodoraceengattung Wachendorfia von vorn her den Bestäubern zugänglich, zugleich aber auch von der Perigonrückseite her für Nicht-Pollinatoren, hier also extranuptial. Das nuptiale Nektarium von Lamium-Arten sezerniert postfloral weiter und wird dann, nunmehr extranuptial, von Ameisen aufgesucht (Sperlich 1939). Die Blütennektarien der Ameisenpflanze Myrmecodia Rumphii werden nach v. d. Pısl (1955) während des Blühens von den Pollinatoren (vermutl. Schwärmern) und später, an den jungen Früchten, von den die Pflanze bewohnenden Ameisen ausgebeutet.

Eine Umwandlung in extranuptiale Nektarien haben die eigentlich nuptialen der Pedaliaceen erfahren. Bestimmte Blütenprimordien entwickeln sich bei einigen Gattungen nicht normal zu Blüten, sondern vergrößern sich unter Wahrung ihrer embryonalen Gestalt lediglich auf 1-2 mm. Nur ihr Discus erlangt Funktionsreife und sezerniert Nektar. Diese Organe finden sich an der Basis der normalen Blüten, aber auch tiefer in der Laubregion. Ähnliche Primordialnektarien sind bei Phaseoleen, der Tiliacee Honckenya, Capparis-Arten und der Sect. Scyphidanthe von Sambucus bekannt (Zimmermand 1.c., v. d. Pijl 1951, Singh 1960, Ihlenfeldt u. Hartmann 1970).

\section{EXTRANUPTIALE NEKTARIEN UND IHRE PROBLEMATIK}

Die Verbreitung der extranuptialen Nektarien geht aus einer noch unvollständigen Liste Zimmerman's hervor, der 69 Familien mit diesen Organen anführt. Die Ansichten über ihre ökologische Bedeutung gehen auch heute auseinander und bedürfen noch besonders der experimentellen Prüfung. Die Umweltrelationen der Laubnektarien lassen sich etwa wie folgt ordnen:

Viele haben keinen ersichtlichen Nutzen im ökologischen Sinne, sind also reine Exkretionsorgane, z.B. nach der ( Saftventil-Theorie » erklärbar. Sie sind oft nur kurzzeitig tätig und an wachsenden Trieben häufig. Als Beispiel seien die fein über die Blattfläche verteilten, einzelnstehenden Drüsenhaare 
mancher Papilionaceen genannt, deren Abscheidungen nur wenig Zucker enthalten, z.T. überhaupt keinen (Schnepf 1965, Bhattacharyya u. MaheshWARI 1971). Bei Vicia drängen sich solche Haare zu scharf umgrenzten, optisch markierten Polstern auf den Stipeln zusammen und erlangen hier auch Umweltbeziehung. Ihre Sekrete sind echte Nektare.

Eine solche organhafte Konzentration ursprünglich diffus verteilter, rein exkretorischer Elemente zu distinkten extrafloralen Nektarien findet sich bei vielen anderen Sippen. Schon innerhalb des vegetativen Bereichs besteht also eine Tendenz zur ökologischen Indienstnahme, manchmal sogar verbunden mit optischer Markierung durch Anthocyanflecke wie z.B. bei Impatiens-, Passiflora- und Grasarten (Andropogon; Bowden 1971, Eragrostis). Ihre reguläre Nutzung durch Insekten, besonders in den Tropen, steht außer Zweifel. Ameisen und Wespen spielen die Hauptrolle, also gerade Kerfe, die als Pollinatoren geringe Bedeutung haben. Die Vorliebe der Wespen, der vermutlichen Stammgruppe der Bienen, für extranuptiale Nektarien ist vielleicht sehr alt und evolutionär bedeutsam. Unter den Bienen verstehen fast nur soziale Apiden den Laubnektar zu nutzen, ohne Zweifel ein abgeleitetes, sekundär erworbenes Verhalten. Die Honigbiene mißachtet gelegentlich die Blüten der Wicken zugunsten ihrer Blattdrüsen. Bei Cassia marilandica (Caesalpiniac.) können diese nach Percival (1965) in Trockenzeiten zu ihrer Hauptnahrungsquelle werden. Die Cassia-Blüten sind nektarlos. Sogar die Nektarien des Adlerfarns werden von Apis besucht.

Die Beziehung der extranuptialen Nektarien zu Ameisen ist von viel größerer Regelmäßigkeit, obwohl auch ihre Symbiosenatur oft in Frage gestellt wird. Unzweifelhaft ist sie z.B. bei den Ameisen-Akazien. Außer von den sog. BeLtschen Körperchen leben die Bewohner vom Nektar der Blattstieldrüsen. Bei Hirtella-Arten (Chrysobalanaceae, aus der Verwandtschaft der Rosaceen) liegen die - denen am Blatt des Kirschbaums vielleicht homologen - Nektarien im Innern blasenförmiger Myrmekodomatien, wo sie von den Insassen ständig beleckt werden.

Nicht ganz so eindeutig ist die myrmekophile Natur der freiliegenden extrafloralen Nektarien, besonders in der europäischen Flora. Daß auch sie von Ameisen besucht werden, ist unbestritten, nur nicht, wieweit der Wirt davon profitiert. Möglicherweise ist die Frage zu eng gestellt. Auch Fremddienlichkeit wäre denkbar, ernähren doch diese Nektarien eine für den Haushalt eines bestimmten Biotops unentbehrliche Tiergruppe, deren Gedeihen indirekt auch dem Nektarspender nützt.

Alle ( Eigennutz-Theorien ) stimmen - von KerNER's unbestätigter Ablenkungstheorie (1879) abgesehen, wonach die extrafloralen Nektarien die Ameisen von den Blütennektarien fernhalten sollen — darin überein, daß sie für eine “ Ameisen-Schutzgarde » gegen Phytophagen plädieren. Gegen diese 
von Delpino und Belt konzipierte “ Myrmekophylaxis " (v. d. Pijl) wird eingewandt, daß die Sekretion oft zu kurzzeitig sei, um Schädlinge wirksam abzuwehren. Nach Schremmer (l.c.) hat z.B. der Adlerfarn ohnehin kaum Feinde; die Imagines eines seiner wenigen Parasiten, einer Blattwespc, profitieren sogar von den Nektarien.

Überzeugendere Varianten der Schutzgarden-Lehre beziehen sich nur auf die Infloreszenzregion, wo auch kurzzeitige Myrmekophylaxis einen Sinn hat. Extranuptiale Drüsen treten hier gehäuft auf. Nach WetTsteIn's Theorie (1888) binden an unreifen Blüten oder Compositenkörbchen tätige Nektarien Ameisen als Schutz gegen Knospenparasiten. Sie ist z.B. durch Versuche von INovye (unveröff., Vortr. XV. Intern. Entom. Congress Washington 1976) an einer Helianthe neuerdings bestätigt worden. Eine zweite - dic erste nicht ausschließende - Deutung gaben Burck (1891) und v. d. PrJl (1951) für die extranuptialen Nektarien vieler von Holzbienen (Xylocopa) besuchter, tropischer Blumen (Thunbergia; Bignoniaceen). Sie nimmt eine Schutzwirkung während der Anthese an. Die angelockten Ameisen vertreiben nachweislich die Bienen, sobald diese den Blütennektar durch Einbruch von außen zu stehlen versuchen. In beiden Fällen schützt die Myrmekophylax is die Reproduktion und hat damit indirekt auch nuptiale Bedeutung.

Eine andersartige, eindeutige Verwendung erfahren extranuptiale Nektarien bei manchen Insektivoren. Der Fangkannendeckel von Sarracenia flava sezerniert reichlich Nektar, der besonders Wespen anzieht, die leicht in die Kanne abstürzen. Ähnlich dürften die Kannenrand-Nektarien von Nepenthes wirken. Insofern sie geflügelte Insekten locken, folgen diese vegetativen Drüsen bereits dem gleichen Prinzip wie die nuptialen, nur mit einem anderen Zweck.

\section{NUPTIALE NEKTARIEN}

Was die nuptialen Nektarien der Blüten betrifft, so ist ihre Motivation so klar eine ökologische, daß ihre eventuelle Stoffwechselfunktion völlig in den Hintergrund tritt. Die früher geäußerte physiologische Erklärung, die Drüsen seien zur Antherendehiszenz nötig (nämlich als wasserentziehende Organe), wird durch die vielen nektarlosen Blüten widerlegt. Die strukturell oft aufwendigen floralen Drüsen, die gegebenenfalls in wenigen Stunden bis zu mehreren ml Nektar austoßen, können auch kaum Exkretionsorgane sein - kommt doch die nicht blühende Pflanze monatelang ohne sie aus. Es gibt allerdings, besonders in jungen Blüten, minimale zuckerhaltige Exsudationen, die von Bestäubern nicht genutzt werden und wohl lediglich interne Gründe haben, blütenbiologisch also keine Nektare sind. Von Daumanv (1970) für etliche Pollenblumen angeführte derartige Abscheidungen (bei Papaver, 
Tulipa, Commelina, Galanthus) sind so zu deuten. Mit den heute verfeinerten Nachweismethoden wird man wohl an vielen Organen derartige Ausschwitzungen feststellen können. Nur in einigen von Pilzmücken bestäubten Blumen (Asarum, Masdevallia-Arten) scheinen sie eine zwar nicht nutritive, aber doch blütenbiologische Bedeutung zu erlangen, indem sie begrenzte Areale der Corolle befeuchten und dadurch die Stimulation der zur Pollination nötigen Eierlegeversuche der Mycetophiliden mitbewirken.

In der Regel ist Blütennektar bekanntlich flüssige Lockspeise für Pollinatoren. Den ältesten Angiospermen fehlte er vermutlich noch ganz. Deren einziges Attractans war Pollen. Das Entstehen der Zwitterblüte muß ja als Konsequenz der von den anemophilen nacktsamigen Vorfahren ererbten Beschränkung dieses späteren Lockmittels auf die Mikrosporophylle angesehen werden, ohne deren gleichzeitige Gegenwart Makrosporophylle keine Attraktion auf Insekten hätten ausüben können. Der primitive Reichtum an Mikrosporangien (Polyandrie) ermöglichte eo ipso den nötigen Ủberschuß an Pollen. Erst der Erwerb nuptialer Nektarien gestattete später die Einsparung und erneute Beschränkung des Pollens auf die Bestäubungsfunktion, d.h. eine - besonders für die Tubifloren und höheren Monokotylen typische - Präzisierung des Blütenapparates. Die Zuwendung des Besuchers wurde von Antheren und Pollen auf die Nektarien abgelenkt. Auch eine teilweise Rückkehr zur Diözie und Diklinie trat ein, da jetzt auch rein weibliche Blüten mit einem Lockmittel, eben den Nektarien, versehen werden konnten.

Auch die Blütennektarien entstanden polyphyletisch und heterogen. Ihre An-und Abwesenheit sowie ihr Bau sind taxonomisch bedeutsame, noch ungenügend verwertete Merkmale. Umfangreiche Sippenkreise führen aus unbekannten Gründen keine nuptialen Nektarien. Wir wissen nicht sicher, ob die Anemophilie der “ Amentiferen » und damit auch ihre Nektarlosigkeit primär oder sekundär ist. Mehrere zoogame, vorherrschend pollenblütige Familien haben vermutlich ab origine kein Nektarium besessen, wie Dilleniaceen, Cochlospermaceen, Ochnaceen, Paeoniaceen, Dipterocarpaceen, Lecythidaceen, Begoniaceen, Melastomataceen, Malpighiaceen, Papaveraceen, Tiliaceen und die Commelinales. Nur ausnahmsweise haben hier einzelne Sippen Nektarien später erworben.

\section{AUS DER VEGETATIVEN REGION “ GELIEHENE " BLÜTENNEKTARIEN}

Einige weitere, notorisch blütennektarlose Familien sind nur durch Einbau extrafloraler Drüsen in den Blütenapparat zu Nektarblumen geworden; so etwa die Marcgraviaceen, deren schon am Laubblatt vorhandenen Drüsen die eigenartigen schlauchförmigen Blütenbrakteen mit Nektar füllen, die den Blüten als Lockorgane beigeordnet sind. Die Nepenthaceen verwenden 
die Äquivalente der vegetativen Kannennektarien auf der Blütenblattfläche zu nuptialem Zweck (Daumann 1930 b, Roth 1954). Sie können dabei nicht verhindern, daß auch Verdauungsenzyme in diesen Nektar eintreten. Auch die trichomatischen Blütennektarien der Malvales sind - mit Ausnahme der Elaeocarpaceen, die einen echten Discus haben - nuptial umfunktionierte vegetative Nektarien, wie sie bei vielen Malvaceen an den Blattstielen auftreten (JANDA 1937). Daß das Blütennektarium der meisten Malvales auf die innere Kelchbasis beschränkt ist, erinnert noch an seine vegetative Herkunft. Es ist nur zwischen den Petalennägeln hindurch zugänglich, weshalb hier Choripetalie zur Notwendigkeit wird. Nur bei einzelnen Tiliaceen (Grewia, Luehea) erlangten auch die Petalen und Staminalröhren die Fähigkeit, derartige Nektarien zu bilden. Bei Gossypium-Arten (Malvac.) hat der Hochblattwirtel des Außenkelches oberseits Nektarien - fast wie im eigentlichen Kelch, jedoch mit noch extranuptialer Funktion. Anscheinend entspricht auch das Kelchsporn-Nektarium der Balsaminenblüte (Impatiens) einem Paar jener gestielten extrafloralen Nektarien, die am Laubblattstiel vieler Arten sitzen (Simon-Moinet 1965). Wohl mehrfach unabhängig sind die Laubdrüsen bei den zoophilen Euphorbiaceen in nuptiale Dienste gestellt worden, wie in den Cyathien der Wolfsmilch.

Vielleicht besaßen die Blüten selbst einmal florale Nektarien, die aber bei vorübergehender Anemophilie samt dem Perianth in Verlust gerieten, dann nach der « DoLLoschen Regel » nicht nochmals am gleichen Ort erscheinen konnten, sich vielmehr durch Hinzuziehung vegetativer Nektarien behelfen mußten. Dabei wird erahnbar, welche wechselvolle Geschichte besonders ein so hochentwickeltes Pseudanthium wie das von Pedilanthus hinter sich haben muß. Erwähnung verdienen auch die Malpighiaceen. Sie haben zwar extranuptiale Nektarien, bei Altweltgattungen sogar an den Kelchblättern, aber keine Nektarblumen. Bei den meisten Neuweltgattungen sind nun anatomisch homologe Kelchdrüsen nuptial geworden, jedoch nicht als Nektarien; sie wandelten sich zu Elaiophoren, d.h. den schon erwähnten ölabscheidenden Drüsen um. Das Öl wird von gewissen Bienen (Anthophoriden) für die Brut gesammelt, indem diese mit den Beinen zwischen den Petalen hindurchgreifen und die Flüssigkeit abschaben. Auch bei einigen Iridaceen und Scrophulariaceen (Cypella; Diascia, Calceolaria) bestehen enge anatomische Beziehungen ölproduzierender Haare zu echten Nektar abscheidenden vegetativen und Blüten-Trichomen verwandter Gattungen, so daß eine gegenseitige Umwandlung anzunehmen ist.

\section{EIGENTLICHE BLÜTENNEKTARIEN}

Die meisten nuptialen Blütennektarien stellen jedoch eine eigenständige, de novo entstandene Apparatur ohne Homologie zu vegetativen Drüsen dar, 
- sofern solche zugleich vorhanden. Z.B. haben die Oleaceen und Convolvulaceen im Laub Trichomnektarien, in der Blüte einen Discus.

Morphologisch lassen sich zwei Hauptgruppen von intrafloralen Nektarien unterscheiden : Phyllom- und Discusnektarien. Nektarphyllome haben ihre Hauptverbreitung bei den Ranales s.l. und Monokotylen. Sie können Blütenhüllblättern wie auch Sporophyllen entsprechen. Am bekanntesten sind die staminodialen Honigblätter der Ranunculaceen (WERTh 1941). Bei den Monokotylen treten sie vor allem als Septalnektarien auf, also als epithelbekleidete Spalten in den Verwachsungsnähten der Fruchtblätter (Daumann 1970). Bei den meisten Centrospermen ist die Staubblattbasis nectarogen (ZaNdonella 1972). Auf den Blütenblättern selbst kommen Nektarien vor allem bei Liliaceen, Orchidaceen und Gentianaceen vor.

Der Discus, noch ausgesprochener eine blüteneigene Erwerbung, ist meist ein perigyner Ringwall oder Gürtel von epithelialem oder mesophyllärem Drüsencharakter; seine morphologische Deutung, d.h. ob er aus Blättern oder Achsenteilen hervorgegangen ist, differiert je nach Verwandtschaftskreis. Er tritt bereits vereinzelt in der Stammgruppe der Dilleniiden (Theaceen, Cistus, Guttiferen) auf. Einen Discus haben die meisten Rosidae (Rosaceen, Leguminosen, Myrtifloren, Rutales) und die Asteridae (Sympetalen). Bemerkenswert ist aber, daß er einigen Sympetalensippen fehlt, die stattdessen offenbar sekundär erworbene Trichomnektarien an der Corolle führen, so die Valerianaceen, Caprifoliaceen, viele Verbenaceen, Lentibulariaceen und manche Scrophulariaceen (Pentstemon, Collinsia, Nemesia).

\section{PROGRESSIONSRICHTUNGEN DER NEKTARIEN-EVOLUTION}

Die nuptialen Nektarien haben innerhalb der Blüte eine Fortentwicklung erfahren, die u.a. durch folgende Progressionen gekennzeichnet ist:

Offenliegende Nektarien wurden in die Tiefe versenkt. Dieser Evolutionsprozess führte, koaptativ zur Verlängerung der Mundorgane entsprechender Bestäuber, zur Einengung des Besucherkreises und damit höherer BestäubungsEffektivität. Die Nektarbergung durch lange Kronröhren und Sporne bot auch besseren Schutz vor Atmosphärilien. Höherentwicklung ist freilich nicht mit Nektarbergung gleichzusetzen, kennen wir doch spezialisierte (eutrope) Blumen mit fast bloßliegendem Nektar wie z.B. Capparis-und Cleome-Arten; diese Lage hat biotechnische Gründe. Nur dadurch, daß die die Blüten bestäubenden Schwärmer durch ihren langen Rüssel gezwungen sind, beim Saugen von den Nektarien Abstand zu halten, kommen sie mit dem Körper in Kontakt mit den weitabstehenden Bestäubungsorganen.

Eine in der Laubregion unerreichte Progression erfuhr die Sekretionskapazität. Sie drückt sich schon in der zytologischen Feinstruktur, aber auch 
in z.T. labyrinthartigen Oberflächenzunahmen der nectarogenen Epithelien aus, wie sie bei Laubnektarien nur andeutungsweise vorkommen (Fagraea, Loganiac.). Die Septaldrüsen der Bromeliaceen z.B. sind wahre « Nektarnieren » (VOGEL 1969a), in die Achse versenkt und gar nicht mehr auf direkte Zugänglichkeit angelegt; das Sekret wird durch enge Poren entleert und tritt dann erst in die eigentliche Nektarkammer ein. Nur mit großen Nektarmengen konnten ja auch Vögel und Fledermäuse als Pollinatoren gewonnen werden.

Eine Progression erfuhr auch die Periodizität der Sekretion, so z.B. ihre Beschränkung auf die Nachtstunden bei Schwärmerblumen oder auf bestimmte Tageszeiten, was zu einer Nischenbildung unter konkurrierenden Nektarblumen führte.

Die Synorganisation von Nektarien mit benachbarten Hilfsorganen war eine weitere Ebene der Evolution. Hierfür gaben schon die Marcgraviaceen ein Beispiel ab. Die Verbergung des Nektars, dieser an sich geruchlosen, chemotaktisch von fern nicht wahrnehmbaren Flüssigkeit, machte die Signalisierung durch Saftmale und Duftmale notwendig. Aus der direkten Wahrnehmung wurde ein Dressurakt durch Assoziation. Die Signale dienen der Nahorientierung und lösen den Rüsselreflex aus. “ Saftdecken 》 in Form von Haaren und Schuppen verhindern die Verdunstung und den Zugriff illegitimer Blütengäste. Überhaupt hat sich die Zuordnung der Blütenteile zum Nektarium biotechnisch vervollkommnet. Sie ist - neben anderen Strukturen des Perianths und neben der Pollenqualität - der Hauptfaktor für die Bestäuberspezifität, z.B. den selektiven Besuch durch bestimmte Solitärbienen, während die relativ gleichförmige Zuckerzusammensetzung des Nektars selbst wenig Einfluß hat. Staunen erregt die Koaptation mancher Nektarien mit einem getrennt gebildeten Behälter. Anders als die meisten Blütensporne sind diejenigen von Corydalis, Viola, Delphinium und manchen Orchideen nektar steril (Vogec 1959, 1969b). Das Sekret rinnt erst von woanders in sie hinein. Bei Disa rosea (Orch.) ragen nectarogene Blütenblatt-Öhrchen in den Kelchsack, während bei anderen Disa-Arten der Saft im Sporne selbst entsteht. In der Linaria-Blüte wird er vom perigynen Discus sezerniert und fließt von dort in einer feinen Kapillarrinne in die Spornspitze, von wo sein Spiegel, ungehindert von Oberflächenspannung oder eingeschlossener Luft, langsam hochsteigt. Die Sekretaufnahme des Besuchers vom Nektarium selbst ist allgemein der primitivere, seine kapillare Fortleitung an einen pollinationstechnisch sinnvolleren Ort der progressive Zustand. Bei Asclepias steigt der Nektar vom Blütenboden durch ein verwickeltes Kanalsystem in die fünf “ Cuculli », die selbst nicht nectarogen sind (GALIL u. ZERoNI 1965). Die lange Crocus-Blütenröhre ist nicht dazu bestimmt, einen Falterrüssel aufzunehmen, sondern leitet kapillar den unter Erdniveau entstehenden Nektar zum Schlund empor, wo ihn Bienen abholen (WERTH 1950), usw. 
Eine Progression der Nektarien besteht auch darin, daß sie an der Arbeitsteilung von Pseudanthien teilnehmen. Manche Blütenverbände differenzieren sich nämlich in « Saftblüten ) und Bestäubungsblüten. In den Köpfen mancher Mimosaceen führt nur eine weitröhrige Zentralblüte ein Nektarium. Besuchende Nachtfalter steuern z.B. bei Calliandra-und Albizzia-Arten daher nur diese an, berühren beim Saugen aber die umliegenden Bestäubungsblüten. Bei der verwandten Parkia haben allein die Blüten eines mittleren Gürtels Nektarien, die distalen besitzen nur Geschlechts-, die basalen Duftfunktion. Die Besucher - Fledermäuse - lecken nur die nectarogene Zone ab. Ähnliches kommt bei Compositen vor. Die weiblichen Körbchen der gynodiözischen Weißen Pestwurz (Petasites albus) führen nur eine einzige zentrale Nektarblüte; sie genügt, um Bestäubung zu sichern, während die übrigen pistillaten Blüten « trocken » sind. Bei der Mutisieen-Gattung Barnadesia (Detpino 1890) liegen im Körbchen-Zentrum drei Nektarblüten, die sich auch durch ihre Weitröhrigkeit von den nectarsterilen Bestäubungsblüten unterscheiden. Teils (B. spinosa, B. rosea) sind sie an den Grund eines regelrechten Körbchen-Schlundes versenkt, teils ( $B$. odorata) münden sie, visuell besonders markiert, in Höhe der übrigen Blüten. Am offenliegenden Spadix der Aracee Spathicarpa sagittifolia liefern die Antherenrudimente der mit den Staubblüten abwechselnden Stempelblüten den Locknektar.

\section{NE KTARIENREDUKTION}

Schließlich sind in größerem Umfang auch Reduktionen von Nektarien aufgetreten, so erstens bei sekundärer Anemophilie innerhalb sonst nectarbürtiger Verwandtschaftskreise wie bei Thalictrum, Chenopodiaceen, Polygonaceen.

Autogame haben zusammen mit dem Abbau sonstiger Lockstrukturen auch ihre Nektarien eingebüßt. Bei Täuschblumen (Da UMand 1971, VogeL 1975) ging die Lockfunktion von echtem Entgelt auf Attrappen über, die allerdings seltener Futter als Brutsubstrat (und Sexualpartner) imitieren. Daher ist die Ophrys-Blüte nektarlos. Das biologische Motiv der " Scheinsaftblumen » von Orchis-Arten ist vielleicht ebenfalls indirekt mit der Partnersuche verknüpft. Die Verödung der Nektarien vieler anderer Orchideen wie Dendrobium, Cattleya, Laelia, Cymbidium ist ökologisch unerklärt. Vielleicht genügt ihnen die Chance gelegentlichen Probierens unerfahrener Hymenopteren.

Auch morphogenetische Ursachen können zum Verschwinden von Nektarien geführt haben. Ein Discus konnte beim Unterständigwerden eines Fruchtknotens gewissermaßen « verschluckt ) werden (Calceolaria) oder ging, wie in manchen Cucurbitaceen-und Papilionaceenblüten, bei der Umstrukturierung des Androeceums verloren. 
Die Rückkehr zur Pollenblütigkeit wie etwa bei der Königskerze oder dem Usambaraveilchen, hat zur Reduktion des Discus geführt, der oft noch angedeutet, aber nicht mehr sekretionsfähig ist. Bei anderen Pollenblumen (Rosa, Mimosa, Acacia pp., Cassia) verschwand er gänzlich.

\section{NUTZUNGSTYPEN DES NEKTARS}

Wir wollen mit einem Hinweis darauf schließen, daß die Nutzung des Blütennektars durch die Bestäuber nicht einheitlich ist. Der Bienenblumennektar dient nicht einmal ausschließlich zu Nährzwecken. Die sog. Feuchtsammler (Apis, Bombus, Meliponiden) benutzen erbrochenen Nektar auch zum Aufkitten der Pollentracht auf die Corbiculae. Diese haben bekanntlich keine Bürstenhaare zum Festhalten des Pollens wie diejenigen der Trockensammler, sondern glatte Pfannen. Die Wespenfamilie Masaridae verwendet Nektar u.a. zum Anrühren des Lehmmörtels für ihre Brutzellen.

Nektartrinken allein zum Stillen des Wasserbedürfnisses wird für manche Vögel angenommen, ebenso bei Tagfaltern, die zu Trockenzeiten besonders eifrige Blütenbesucher sind. Sonst aber wird Nektar direkt oder indirekt als Nahrungsstoff konsumiert. Jedoch dient er auch als solcher nicht immer als Lockmittel und Belohnung. Die Nektarien im Innern mancher Kesselfallenblumen haben eine ganz andere Funktion. Sie ermöglichen nur das Überleben der gefangenen Insassen für bestimmte Zeit, dienen der Verproviantierung im unfreiwilligen Asyl, wie z.B. die Narben flüssigkeit von Arum (Daumann 1930a), das Sekret der sterilen PBlüten von Cryptocoryne Griffithii, die trichomatischen Nektarienpolster im Kessel vieler Aristolochien.

Auch als Attractans kann Nektar einen verschiedenen ethologischen Stellenwert besitzen, je nachdem er offenliegt und direkt als Futter wahrgenommen wird, oder verborgen und nur durch Assoziation mit Signalen, d.h. einen Lernakt, zu finden ist. Offen abgeschiedenes Sekret z.B. in napfförmigen Blüten wird von den darüber hinweglaufenden Dipteren anhand des Feuchtglanzes und durch Betasten direkt erkannt. Der Nektar kann “ Straßen ” bilden, auf denen die ihn aufnippenden Fliegen an die Bestäubungsorgane herangeleitet werden (z.B. bei Periploca) oder auch Flächen, die an den Rand einer Absturzzone von Kesselfallen führen (Aristolochia durior, Cryptocoryne ciliata).

Alle Dipteren und Käfer nehmen den Nektar nur zu ihrer Eigenernährung auf. Dies gilt auch für die Tag-und Nachtfalter, Fledermäuse und Vögel (lediglich von Kolibris ist bekannt, daß sie auch ihre Jungen mit regurgitiertem Nektar füttern). Die betreffenden Blumen sind also im wesentlichen « Direkternährer ». Im Gegensatz hierzu stellen die Bienenblumen in erster Linie Nahrungsquellen für Brutpflege und soziale Stockversorgung dar, wozu ja auch 
der eingesammelte Pollen dient. Nur einen kleinen Teil des Sekretes resorbieren die Bienen aus dem Honigmagen für sich selbst, der größte Teil kommt, zu Honig verarbeitet, dem Larvenproviant zugute. Bei den sozialen Bienen wandert er unter Weitergabe an die Stockgenossen in die Honigtöpfe und Honigzellen zur Versorgung und Überwinterung des Volkes. Diese indirekte, nutritive Verwendung teilt der Bienenblumennektar mit einer zweiten, vegetabilischen Zuckerquelle der Bienen und Ameisen : mit dem durch Vermittlung der Blattläuse entstehenden Honigtau.

Eingegangen im Mai 1977. Reçu pour publication en mai 1977.

\section{RÉSUMÉ}

Cette revue résume nos connaissances actuelles sur les divers aspects des nectaires et les questions qui restent en suspens : morphologie, topographie, répartition dans le règne végétal et importance écologique, ainsi que les nectaires extra-floraux et quelques cas exceptionnels. On discute également des directions de l'évolution des nectaires et des divers moyens par lesquels les animaux utilisent le nectar.

\section{LITERATUR}

Bhattacharyya B., Maheshwari J. K., 1971. - Studies on extrafloral nectaries of the Leguminales. I : Papilionaceae. Proc. Indian nat. sci. Acad., B, 37, 11-30.

Bowden B., 1971. - Studies on Andropogon gayanus. VI : The leaf nectaries of A. gayanus var. bisquamulatus (Hochst). Hack. (Gramineae). Bot. J. Linn, Soc., 64, 77-80.

Brown W., 1938. - The bearing of nectaries on the phylogeny of flowering plants. Proc. am. philosoph. Soc. Philadelphia, 79, 549-595.

Burck W., 1891. - Beiträge zur Kenntnis der myrmekophilen Pflanzen und der Bedeutung der extranuptialen Nektarien. Ann. Jard. Bot. Buitenzorg, 10, 73-144.

Caspary J., 1848. - De nectariis. Dissert. Elberfeld.

Daumans E., 1930a. - Nektarabscheidung in der Blütenregion einiger Araceen... Planta (Berlin), 12, 38-48.

Daumanx E., 1930b. -- Das Blütennektarium von Nepenthes. Beih. Bot. Zentralblatt Abt. I, 47, 1-40.

Daumann E., 1970. - Das Blütennektarium der Monokotyledonen unter besonderer Berücksichtigung seiner systematischen und phylogenetischen Bedeutung. Fedde's Repert., 80, 436-590.

Daumanx E., 1971. - Zum Problem der Täuschblumen. Preslia (Prag), 43, 304-317.

Delpino F., 1890. - Note ed osservazioni botaniche, dec. seconda. Malpighia, 4, 3-34.

Feldhofer E., 1933. - Beiträge zur physiologischen Anatomie der nuptialen Nektarien aus den Reihen der Dicotylen. Dissert. München.

GaLil J., Zeroni M., 1965. - Nectar system of Asclepias curassavica. Bot. Gazette, 126, 144-148.

Ihlenfeld H. D., Hartmann H., 1970. - Die Gattung Harpagophytum (Burch.) DC ex Meissn. Mitt. Staatsinst. allg. Bot. Hamburg, 13, 15-69.

JANDA Ch., 1937. - Die extranuptialen Nektarien der Malvaceen. Österr. Bot. Z., 86, 81-130.

Kerner A., 1879. - Die Schutzmittel der Blüten gegen unberufene Gäste. 2. Aufl.- Innsbruck, Wagner. 
Knuth P., 1904. - Handbuch der Blütenbiologie III, 1. - Leipzig, Engelmann.

Percival M., 1965. - Floral biology. - Oxford, London, Pergamon Press.

Rотн I., 1954. - Entwicklung und histogenetischer Vergleich der Nektar- und Verdauungsdrüsen von Nepenthes. Planta, 43, 361-378.

SCHNEPF E., 1965. - Licht- und elektronenmikroskopische Beobachtungen an den TrichomHydathoden von Cicer arietinum. Z. Pflanzenphysiol., 53, 245-254.

Schremmer F., 1969. - Extranuptiale Nektarien. Beobachtungen an Salix eleagnos Scop. und Pteridium aquilinum. Österr. Bot. Z., 117, 205-222.

Simon-Moinet L., 1965. - Homologies foliaires et florales chez l'Impatiens balsamina L. Recherches préliminaires. C.R., 260, 2047-2050.

Singr S., 1960. - Morphological studies in some members of the family Pedaliaceae I : Sesamum indicum Oc. Phytomorphology, 10, 65-81.

SPERLich A., 1939. - Excretionsgewebe in : K. Linsbauer (Herausgeb.), Handbuch der Pflanzenanatomie Bd IV Lief. 38, Berlin, Borntraeger.

VAN Der PiJl L., 1951. - On the morphology of some tropical plants : Gloriosa, Bougainvillea, Honckenya and Rottboelia. Phytomorphology, 1, 185-188.

VAN der PiJl L., 1955. - Some remarks on Myrmekophytes. Phytomorphology, 5, 190-200.

Vogel St., 1959. - Organographie der Blüten kapländischer Ophrydeen mit Bemerkungen zum Koaptationsproblem. Abh. Akad. Wiss. u. Lit. Mainz, math-.nat. Kl. 6 u. 7, 267-532.

Vogel St., 1967. - Iris fulva Ker-Gawl., eine Kolibriblume. Dtsch. Iris- und Lilienges., 1-11.

VogEL St., 1969 a. - Chiropterophilie in der neotropischen Flora. Neue Mitteilungen III. Flora, Abt. B, 157, 289-323.

VOGEL St., 1969b. - Über synorganisierte Blütensporne bei einigen Orchideen. Österr. Bot. $Z$., 116, 244-262.

Vogel. St., 1974. - Ölblumen und ölsammelnde Bienen. Reihe trop. und subtrop. Pflanzenwelt 7. Abh. Akad. Wiss. u. Lit. Mainz., math. u. nat. Kl., 284-547

Vogel St., 1975. - Mutualismus und Parasitismus in der Nutzung von Pollenträgern. Verh. dtsch. Zool. Ges., 1975, 102-110.

Werth E., 1941. - Die Blütennektarien der Ranunculaceen und ihre phylogenetische Bedeutung. Ber. dtsch. Bot. Ges., 59, 246-256.

Werth E., 1950. - Crocus und Colchicum, zwei blütenbiologische Paradoxa. Ber. dtsch. Bot. Ges., 63, 83-88.

Wettstein R. von, 1888. - Über die Compositen der österreichisch-ungarischen Flora mit zuckerabscheidenden Hüllschuppen. Sitz. Ber. Akad. Wiss. Wien, 97, Abt. I, $578 f$.

Zandonelua P., 1967. - Stomates des nectaires floraux chez les Centrospermales. Bull. Soc. bot. Fr., 114, 11-20.

Zandonella P., 1972. - Le nectaire floral des Centrospermales. Thèse Univ. Lyon.

Zimmermann J., 1932. - Über die extrafloralen Nektarien der Angiospermen. Beih. Bot. Centralbl., Abt. I, 49, 99-196. 